\title{
Human Health and Ecological Risk Assessment of Two Common Antibiotics in Drinking Source Water
}

Hanyu Ju, Jiquan Zhang*, Caiyun Sun, Sijia Li

School of Environment, Northeast Normal University, Institute of Natural Disaster Research, Northeast Normal University, Changchun 130024, China

\section{饮用水源地两种抗生素的生态风险评价和人体健康风 险评价}

鞠含俞, 张继权 ${ }^{*}$, 孙彩云, 李思佳

东北师范大学环境科学学院, 长春 130024 中国

\begin{abstract}
Take the sulfamethoxazole and metronidazole as two examples, exploring the spatial distribution of antibiotics in two drinking water sources, which are in Xin Licheng reservoir and Shitou Koumen reservoir, and evaluating ecological risk and human health risk. The average concentration of sulfamethoxazole and metronidazole which sampling in the sampling points of Xin Licheng reservoir are $0.05936\llcorner\mathrm{~g} / \mathrm{L}$ and $0.05183 \mathrm{Lg} / \mathrm{L}$, and that in the sampling points of Shitou Koumen reservoir are $0.03487 ц \mathrm{~g} / \mathrm{L}$ and $0.04244 \mathrm{Lg} / \mathrm{L}$. The concentration of antibiotics in Xin Licheng reservoir is more than that in Shitou Koumen reservoir, and concentration of antibiotics in aquaculture water is more than others. After calculating, the entropy of sulfamethoxazole and metronidazole is less than threshold, so the two kinds of antibiotics in two drinking water sources are no ecological and human health risks.
\end{abstract}

Key word: antibiotic; drinking water source; ecological risk; human health risk

\section{摘要}

以磺胺甲恶唑和甲硝唑两种常见抗生素为例, 探究长 春两处饮用水源地新立城水库和石头口门水库抗生 素的空间分布以及生态风险和人体健康风险。磺胺甲 恶唑和甲硝唑在新立城水库和石头口门水库的各采

*通讯作者: zhangjq022@nenu.edu.cn
样点均有检出其平均浓度分别为 $0.05936 \mathrm{~L} / \mathrm{L}$ 、 $0.05183 ц \mathrm{~g} / \mathrm{L}$ 和 $0.03487 ц \mathrm{~g} / \mathrm{L} 、 0.04244 Ц \mathrm{~g} / \mathrm{L}$ 。新立 城水库抗生素浓度明显高于石头口门水库, 并且水产 养殖水域内抗生素浓度明显偏高。经计算磺胺甲恶唑 和甲硝唑的风险熵值均低于阈值, 两种抗生素在两处 饮用水源地均无生态风险和人体健康风险。

关键词: 抗生素 饮用水源地 生态风险 人体健康风 险

\section{1. 引言}

随着检测技术的不断发展, 人们对痕量污染物的环境 残留问题日益重视。作为痕量污染物的一类典型代表, 抗生素是一类自然或半人工合成的有机物, 并广泛应 用于各类畜牧业、水产养殖业以及种植业, 其作用而 被广泛关注。有机体对抗生素的利用率较低, 通常有 80\%-90\%的抗生素及其代谢产物（仍具有药物活性） 通过尿液和粪便被排放到自然环境中[1]。研究表明, 在地表水、地下水、土壤以及沉积物等各类环境介质 中均检测出抗生素残留 [2]。而抗生素残留所导致的 抗性基因的产生是其最主要影响之一[3], 这直接威 胁着此环境介质周围居民的健康以及此区域内的生 态安全。

水库是与人类日常生活链接最紧密的一类环境 介质[4], 是附近居民的饮用水源地, 为其周围居民 饮水、农田灌溉以及养殖业用水提供支持 [5], 并且 对于其周围的生态安全以及附近居民的人体健康产 生重要影响。因此, 对于水库的生态风险评价以及人 体健康风险评价都有着十分重要的意义。

本研究将以长春市的两个主要饮用水源地 (石 头库门水库和新立城水库) 为例, 选取两种常见的抗 
Risk Analysis and Crisis Response in Big Data Era (RAC-16)

生素, 磺胺甲恶唑 (SMZ) 和甲硝唑 (MTZ), 对其周 围环境生态以及以水库作为饮用水源地的居民的人 体健康进行风险评价, 为后续抗生素在水库及地表水 中的污染风险管控提供理论依据。

\section{2. 材料与方法}

\section{1. 水样采集}

2016 年 5 月, 对新立城水库和石头口门水库进行采 样。根据两个水库的各类水文特征进行布点, 对新立 城水库布 8 个采样点, 石头口门水库布 9 个采样点, 如图 1 所示。水样采集与保存程序符合国家标准 ( GB/T 5750.2-2006)。各采样点均采集 $1 \mathrm{~L}$ 水样, 实验 前保存于 $4^{\circ} \mathrm{C}$ 条件下, 采样结束后一周内完成实验。

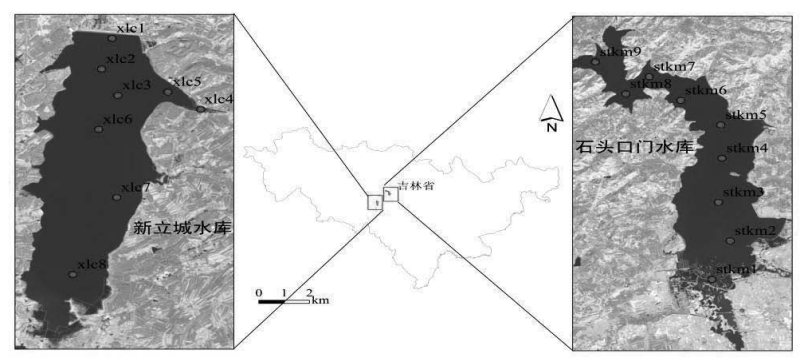

\section{2. 仪器与试剂}

仪器: 高效液相色谱仪 (Waters 公司)、C18 色谱柱 ( $2.1 \mathrm{~mm} \times 50 \mathrm{~mm}, 5 \mu \mathrm{m}$, 美国 Waters 公司)、菼 光检测器 2489 型 (Waters 公司)、电导检测器 2475 型 (Waters 公司) 、旋转蒸发器、12 孔固相萃取装置。 试剂: 标准品磺胺甲恶唑（纯度为 $99.0 \%$ ）和甲硝唑 （纯度为 99.5\%）均购自德国 Dr.Ehrenstorfer GmbH 公司。固相萃取柱为 Waters 公司 HLB 固相萃取柱。 Na2EDTA 粉末 (纯度 $\geqslant 99 \%$ ), 甲醇、甲酸及乙酸乙 酯均为色谱纯。实验用水为超纯水。

\section{3. 样品预处理}

将 $1 \mathrm{~L}$ 水样经 $0.45 \mathrm{~L} \mathrm{~m}$ 滤膜过滤, 置于大烧杯中, 依 次向其中加入 $0.4 \mathrm{ml}$ 浓盐酸和 $0.5 \mathrm{gNa}$ EEDTA 粉末, 摚 拌至完全溶解。将 HLB 固相萃取柱分别用 $6 \mathrm{ml}$ 乙酸 乙酯-甲醇 $(\mathrm{V}: \mathrm{V} / 1: 1)$ 溶液、 $6 \mathrm{ml}$ 甲醇溶液和 $6 \mathrm{ml}$ 酸 性水 (甲酸调 $\mathrm{PH}=2-3$ ) 对其进行活化。再将 $1 \mathrm{~L}$ 水样 以不超过 $15 \mathrm{ml} / \mathrm{min}$ 的速度进行固相萃取（整个过程 防止柱子干燥)。待水样全部通过固相萃取柱, 先对 柱子进行干燥, 柱子完全干燥后用大于 $6 \mathrm{ml}$ 的 $5 \%$ 的 甲醇水溶液淋洗, 去除基质干扰。最后用甲醇对固相 萃取柱中的污染物进行洗脱, 收集于干净的雉形瓶中, 并对其进行旋干（旋干温度应不高于 $30^{\circ} \mathrm{C}$ 以防止抗 生素分解), 最后用甲醇定容至 $1.5 \mathrm{ml}$ 与上机瓶中液 相色谱上机使用。

\section{4. 水中抗生素的测定}

采用高效液相色谱法测定水样中抗生素的浓度, 回收 率为 $81.90 \%-110.00 \%$, 相对标准偏差为 1.80\%-7.10\%。 2.5. 生态风险评价方法
本论文采用熵值法对水中抗生素进行生态风险评价, 计算公式如下:

$$
\begin{aligned}
& \mathrm{RQ}=\frac{\mathrm{MEC}}{\mathrm{PNEC}} \\
& \mathrm{RQ}_{\text {总 }}=\sum \mathrm{RQ}_{\mathrm{i}}
\end{aligned}
$$

式中: $R Q$ 为风险熵值, 无量纲; $M E C$ 为抗生素在实 际水体中的测量浓度 $\mathrm{ng} \cdot \mathrm{L}^{-1}$; PNEC 为预测无效应浓度, 是在现有认知下不会对环境中微生物或生态系统产 生不利效应的最大药物浓度, $\mathrm{ng} \cdot \mathrm{L}^{-1}$ (磺胺甲恶唑和 甲硝唑的 PNEC 值分别为 $1740 \mathrm{ng} \cdot \mathrm{L}^{-1}$ 和 $\left.1300 \mathrm{ng} \cdot \mathrm{L}^{-1}\right)[6]$; $R Q$ 总为水体中各类抗生素的联合风险摘值, 无量纲; $\mathrm{RQ}_{\mathrm{i}}$ 为某一种抗生素的风险摘值, 无量纲。

根据所得 $R Q$ 值可评价出该类污染物做出相应的生态 风险评价。当 $0.01 \leqslant R Q<0.1$, 则认定为低风险或无 风险; 当 $0.1 \leqslant R Q<1$, 为中等风险; 当 $R Q \geqslant 1$, 则 存在高风险[7]。又因为水体中可能存在多种抗生素, 因此其对生态造成的风险也是可叠加的, 因此本研究 采用叠加模式对多种抗生素在该生态环境中的风险 进行评价。

\section{6. 人体健康风险评价方法}

本研究通过饮用水和皮肤暴露两种暴露途径对水体 中抗生素进行人体健康风险评价。评价模型采用 USEPA 的人体健康风险评价模型, 计算公式如下 [8]:

$$
\mathrm{LADD}_{\mathrm{w}}=(\mathrm{C} \times \mathrm{IR} \times \mathrm{EF} \times \mathrm{ED}) /(\mathrm{BW} \times \mathrm{AT})
$$

$\mathrm{LADD}_{\mathrm{d}}=(\mathrm{C} \times \mathrm{SA} \times \mathrm{Kp} \times \mathrm{ET} \times \mathrm{EF} \times \mathrm{ED} \times \mathrm{CF}) /(\mathrm{BW} \times \mathrm{AT})$

$$
\mathrm{HI}=\frac{\mathrm{LADD}}{\mathrm{RfD}}
$$


Risk Analysis and Crisis Response in Big Data Era (RAC-16)

$$
\begin{gathered}
\mathrm{RfD}_{\mathrm{d}}=\mathrm{RfD}_{\mathrm{w}} \times \mathrm{ABSg} \\
\mathrm{HI}_{\text {总 }}=\sum \mathrm{HI}_{\mathrm{i}}
\end{gathered}
$$

式中, $L A D D_{w}$ 为抗生素通过饮用水的暴露计量, $\mu$ $\mathrm{g} \cdot \mathrm{kg}^{-1} \cdot \mathrm{d}^{-1} ; \mathrm{LADD}_{\mathrm{d}}$ 为饮用水通过皮肤的暴露计量, $\mu$ $\mathrm{g} \cdot \mathrm{kg}^{-1} \cdot \mathrm{d}^{-1} ; \mathrm{C}$ 为实际水体中抗生素的浓度, $\mu \mathrm{g} \cdot \mathrm{L}^{-1} ; \mathrm{H}$ 为每日饮水量, $L \cdot d^{-1} ; E F$ 为暴露频率, day year ${ }^{-1}$; ED 未暴露持续时间, year; $\mathrm{BW}$ 为体重, $\mathrm{Kg}$; $\mathrm{AT}$ 为平均 暴露时间, 为 ( $E D \times 365)$, day; SA 为皮肤暴露面积, $\mathrm{cm}^{2} ; \mathrm{Kp}$ 为皮肤渗透系数, $\mathrm{cm} \cdot \mathrm{h}^{-1} ; \mathrm{ET}$ 人类通过洗澡 而造成的皮肤暴露时间, $h \cdot d a y^{-1}$; CF 为单位转换系数,
$L \cdot(1000 \mathrm{~cm})^{-1} ; \mathrm{HI}$ 为危害熵值, 无量纲; RfD 为某 一污染物的非致癌暴露参数, $\mu \mathrm{g} \cdot \mathrm{kg}^{-1} \cdot \mathrm{d}^{-1} ; \mathrm{RfD}_{\mathrm{w}}$ 为通 过直接食用暴露途径的暴露参数,

$\mu \mathrm{g} \cdot \mathrm{kg}^{-1} \cdot \mathrm{d}^{-1}$ (磺胺甲恶唑和甲硝唑的 $\mathrm{RfD}_{\mathrm{w}}$ 值分别 为 $130 \mu \mathrm{g} \cdot \mathrm{kg}^{-1} \cdot \mathrm{d}^{-1}$ 和 $2 \mu \mathrm{g} \cdot \mathrm{kg}^{-1} \cdot \mathrm{d}^{-1}$ ) [9]; $\mathrm{RfD}_{\mathrm{d}}$ 为通过皮 肤暴露途径的暴露参数, $\mu \mathrm{g} \cdot \mathrm{kg}^{-1} \cdot \mathrm{d}^{-1}$; $\mathrm{ABSg}$ 为肠胃吸 收系数, 无量纲; $\mathrm{HI}$ 总为各类抗生素对人体健康的风 险熵值总和, 无量纲; $\mathrm{HI}_{\mathrm{i}}$ 为某种抗生素对人体健康 色风险熵值, 无量纲。式中所用的各类参数如表 1 所示[8][10]:

\begin{tabular}{ccc}
\multicolumn{3}{c}{ 表 1.各类参数的参考值 } \\
\hline 定义 & 单位 & 数值 \\
\hline $\mathrm{HI}^{1}$ & $\mathrm{~L} \cdot \mathrm{d}^{-1}$ & 1.41 \\
$\mathrm{EF}_{\mathrm{w}}{ }^{1}$ & day $\cdot \mathrm{year}^{-1}$ & 365 \\
$\mathrm{EF}_{\mathrm{d}}{ }^{1}$ & day $\cdot \mathrm{year}{ }^{-1}$ & 360 \\
$\mathrm{ED}^{1}$ & $\mathrm{year}$ & 73.65 \\
$\mathrm{BW}^{1}$ & $\mathrm{Kg}$ & 53.6 \\
$\mathrm{SA}^{1}$ & $\mathrm{~cm}{ }^{2}$ & 20091 \\
$\mathrm{Kp}^{1}$ & $\mathrm{~cm} \cdot \mathrm{h}^{-1}$ & 0.2 \\
$\mathrm{ET}^{1}$ & $\mathrm{~h} \cdot \mathrm{day}^{-1}$ & 0.25 \\
$\mathrm{ABSg}^{2}$ & - & 0.5 \\
\hline
\end{tabular}

'(Expose Factors Handbook of China Population);

$$
{ }^{2} \text { (USEPA 1989). }
$$

由以上公式及参数即可计算出人体健康的风险商值。经检测得, 磺胺甲恶唑和甲硝唑两种抗生素在新立城 考虑到敏感人群将风险阈值设定为 0.1 , 当 $\mathrm{HI} \geqslant 0.1$ 水库和石头口门水库的各采样点均被检出其分布特 则认为存在健康风险[11]。

\section{点如表 2 所示。}

两种抗生素在新立城水库和石头口门水库各采

3. 结果与讨论 样点的分布如图 2. 所示。

\begin{tabular}{|c|c|c|c|c|}
\hline & $\operatorname{Max}$ & Mean & Min & $\mathrm{CV} \%$ \\
\hline & 新立城 & 新立城 & 新立城 & 新立城 \\
\hline SMZ (ц g/L) & 0.12897 & 0.05936 & 0.02586 & 58.50506 \\
\hline \multirow[t]{2}{*}{ MTZ (ц g/L) } & 0.10839 & 0.05183 & 0.03063 & 49. 89612 \\
\hline & 石头口门 & 石头口门 & 石头口门 & 石头口门 \\
\hline $\operatorname{SMZ}(ц \mathrm{~g} / \mathrm{L})$ & 0.04794 & 0.03487 & 0.02286 & 19. 11294 \\
\hline MTZ (ц g/L) & 0.06292 & 0.04244 & 0.03121 & 21. 48431 \\
\hline
\end{tabular}

\section{1. 水体中抗生素的检测与空间分布}

表 2. 两种抗生素在水库中的浓度分布 
Risk Analysis and Crisis Response in Big Data Era (RAC-16)
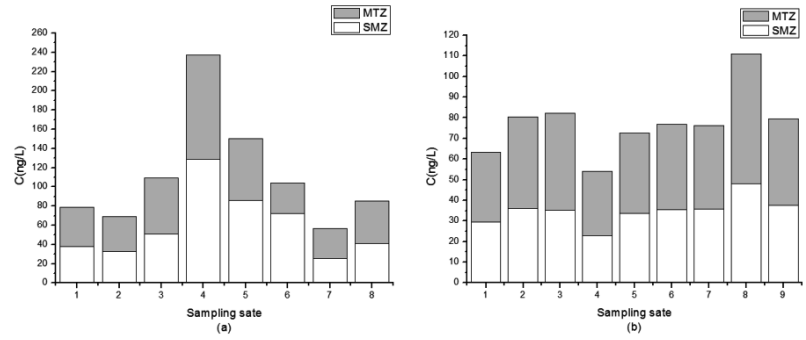

图 2.两种抗生素在新立城水库和石头口门水库的空间分布

由图可见两种抗生素的浓度变化趋势相同。由 此可见，两种抗生素的污染源是相同。新立城水库在 采样点 4 处两种抗生素浓度最高, 石头口门水库在采 样点 8 处取得最高浓度。并且在采样时发现在新立城 水库采样点 4 处有一小型水产养殖基地。由此可见, 水产养殖是水体中抗生素的一个主要的污染源。

\section{2. 生态风险评价}

运用熵值法对磺胺甲恶唑和甲硝唑两种抗生素进行 生态风险评价, 评价结果如表 3 所示。
由表可知, 磺胺甲恶唑和甲硝唑两种抗生素在两个水 库中均含有较低或无生态风险。但根据数据仍可观察 到, 新立城水库的风险熵值要高于石头口门水库, 因 此新立城水库的生态风险要高于石头口门水库, 这也 应引起管理部门的重视。

\section{3. 人体健康风险评价}

对两个水库两种抗生素的人体健康风险评价结果如 表 4.所示。

表 3. 水体中 SMZ 和 MTP 的风险熵值

新立城水库

\begin{tabular}{|c|c|c|c|c|c|c|c|c|}
\hline & RQ 最小值 & RQ 最大值 & $\begin{array}{c}\mathrm{RQ} \text { 最小 } \\
\text { 值 }>1\end{array}$ & $\begin{array}{c}1>\mathrm{RQ} \text { 最 } \\
\text { 小值 }> \\
0.1\end{array}$ & $\begin{array}{l}\mathrm{RQ} \text { 最小 } \\
\text { 值 }<0.1\end{array}$ & $\begin{array}{c}\mathrm{RQ} \text { 最大 } \\
\text { 值 }>1\end{array}$ & $\begin{array}{c}1>\mathrm{RQ} \text { 最 } \\
\text { 小值 }> \\
0.1\end{array}$ & $\begin{array}{l}\mathrm{RQ} \text { 最小 } \\
\text { 值 }<0 \text {. }\end{array}$ \\
\hline 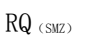 & 0.01486 & 0.07412 & & & . & & & . \\
\hline $\mathrm{RQ}(\mathrm{arP})$ & 0.02356 & 0. 08337 & & & . & & & . \\
\hline $\mathrm{RQ}_{\text {(总) }}$ & 0.03842 & 0.15749 & & & . & & . & \\
\hline \multicolumn{9}{|c|}{ 石头口门水库 } \\
\hline & $R Q$ 最小值 & RQ 最大值 & $\begin{array}{c}\mathrm{RQ} \text { 最小 } \\
\text { 值 }>1\end{array}$ & $\begin{array}{l}\mathrm{RQ} \text { 最小 } \\
\text { 值 }>0.1\end{array}$ & $\begin{array}{l}\mathrm{RQ} \text { 最小 } \\
\text { 值 < } 0.1\end{array}$ & $\begin{array}{c}\mathrm{RQ} \text { 最大 } \\
\text { 值 }>1\end{array}$ & $\begin{array}{l}\mathrm{RQ} \text { 最大 } \\
\text { 值 }>0.1\end{array}$ & $\begin{array}{l}\mathrm{RQ} \text { 最大 } \\
\text { 值 }<0 .\end{array}$ \\
\hline 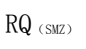 & 0.01314 & 0.02755 & & & . & & & . \\
\hline $\mathrm{RQ}$ & 0.02401 & 0.0484 & & & . & & & . \\
\hline $\mathrm{RQ}_{\text {(总) }}$ & 0.03715 & 0.07595 & & & . & & & . \\
\hline
\end{tabular}


Risk Analysis and Crisis Response in Big Data Era (RAC-16)

\section{表 4.水体中 SMZ 和 MTZ 的人体健康风险摘值}

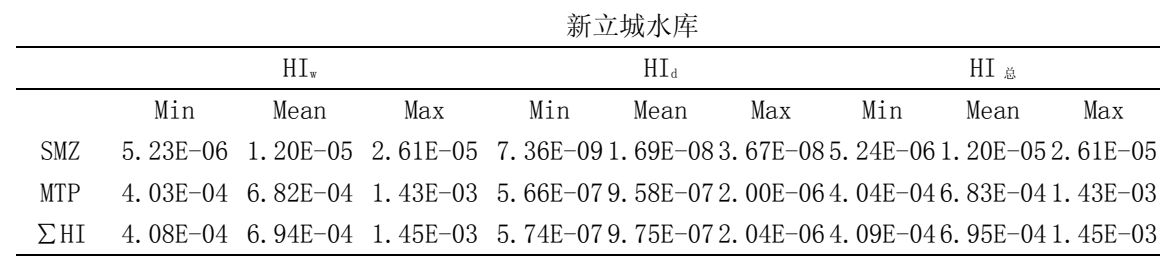

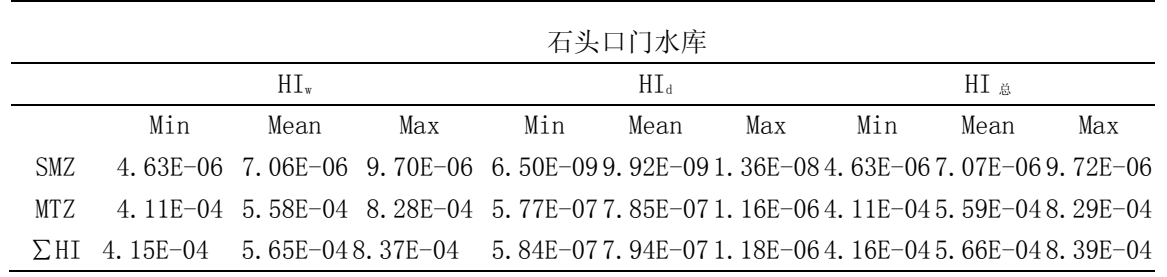

由上表可知以上抗生素通过各类途径对人体 均无健康风险, 其健康风险熵值总均低于 0.1 , 因此磺胺甲恶唑和甲硝唑在新立城水库和石 头口门水库均未对人体健康存在风险。但从上 表可以看出 MTZ 的风险商值要远大于 $S M Z$, 因此相关部门应加强对 MTZ 环境中的排放管 控。

\section{4. 结论}

本研究选用磺胺甲恶唑和甲硝唑两种常见的 抗生素进行测定评价, 最终在两个水库共 17 个采样点均被检出, 这足以显示这两种抗生素 在水体中是广泛存在的。并且通过数据分析可 得靠近水产养殖的水域抗生素明显高于其他 水体, 因此应加强对试产养殖业抗生素排放的 管控。研究发现所选定的两种抗生素在所研究 区域内虽未存在生态风险和人体健康风险, 但 新立城水库的风险熵值明显高于石头口门水 库。因此, 管理部门应加强对新立城水库及附 近环境的抗生素类药物的管理和排放。

\section{Acknowledgement}

This study on national major water projects (2014ZX07201-011) and the National Non-Profit Research Program of China (No.20141015) for the project support.

\section{致谢}

本研究以国家重大水专 (2014ZX07201-011) 和水利部公益性行业科研专项经费项目 (201401015) 为项目支持。

\section{参考文献}

[1] Yan C, Yang Y, Zhou J, et al. Antibiotics in the surface water of the Yangtze Estuary: occurrence, distribution and risk assessment. Environmental Pollution, 2013, 175: 22-29.

[2] Hu X, Zhou Q, Luo Y. Occurrence and source analysis of typical veterinary antibiotics in manure, soil, vegetables and groundwater from organic vegetable bases, northern China. Environmental Pollution, 2010, 158(9): 2992-2998.

[3] Isidori M, Lavorgna M, Nardelli A, et al. Toxic and genotoxic evaluation of six antibiotics on non-target organisms. Science of the total environment, 2005, 346(1): 87-98.

[4] Kadri F. The Assessment of Risk Caused by Fire and Explosion in Chemical Process Industry: A Domino Effect-Based Study. Journal of Risk Analysis and Crisis Response. 3(2): 66-76, 2013.

[5] Sun C, Zhang J, Ma Q, et al. Human Health and Ecological Risk Assessment of 16 Polycyclic Aromatic Hydrocarbons in Drinking Source Water from a Large Mixed-Use Reservoir. International journal of environmental research and public health, 2015, 12(11): 13956-13969.

[6] Jiang Y, Li M, Guo C, et al. Distribution and ecological risk of antibiotics in a 
Risk Analysis and Crisis Response in Big Data Era (RAC-16)

typical effluent-receiving river (Wangyang River) in north China. Chemosphere, 2014, 112: 267-274.

[7] Vryzas Z, Alexoudis C, Vassiliou G, et al. Determination and aquatic risk assessment of pesticide residues in riparian drainage canals in northeastern Greece. Ecotoxicology and environmental safety, 2011, 74(2): 174-181.

[8] EPA A B D. Risk Assessment Guidance for Superfund. Volume I: Human Health Evaluation Manual (Part A)[R]. EPA/540/1-89/002, 1989.

[9] Schwab B W, Hayes E P, Fiori J M, et al. Human pharmaceuticals in US surface waters: a human health risk assessment. Regulatory Toxicology and Pharmacology, 2005, 42(3): 296-312.

[10] 保护部. 中国人群暴露参数手 册 (成人 卷). 2013 .

[11] 成兴源. 城市污水处理厂出水用于绿化 灌溉时全氟辛酸健康风险评价. 兰州交 通大学, 2013. 